\title{
Hypothesis
}

\section{A docking model of key components of the DISC complex: death domain superfamily interactions redefined}

\author{
Christian H. Weber, Claudius Vincenz* \\ Department of Pathology, The University of Michigan Medical School, Ann Arbor, MI 48109-0602, USA
}

Received 14 November 2000; revised 22 January 2001; accepted 22 January 2001

First published online 26 February 2001

Edited by Gunnar von Heijne

\begin{abstract}
Apoptosis is mediated by a highly regulated signal transduction cascade that eventually leads to precisely directed cell death. The death-inducing signaling complex (DISC), composed of Fas, FADD, and caspase-8, is an apical signaling complex that mediates receptor-induced apoptosis. We have docked the experimentally determined structures of the Fas and FADD death domains into a model of a partial DISC signaling complex. The arrangement of Fas and FADD was determined using the interaction modes of the two heterodimer crystal structures determined to date, Pelle/Tube and Apaf-1/procaspase-9. The proposed model reveals that both interactions can be accommodated in a single multimeric complex. Importantly, the model is consistent with reported site-directed mutagenesis data indicating residues throughout the domain are critical for function. These results imply that members of the death domain superfamily have the potential for multivalent interactions, offering novel possibilities for regulation of apoptotic signaling. (C) 2001 Federation of European Biochemical Societies. Published by Elsevier Science B.V. All rights reserved.
\end{abstract}

Key words: Docking model; Apoptosis; Death domain superfamily; Caspase recruitment domain; Death effector domain; Death domain

\section{Introduction}

Apoptosis, or programmed cell death, is a vital cell lifecycle decision point in multicellular organisms. It is a necessary event in normal embryogenesis and development, maintenance of homeostasis, and immune system function [1]. Consistent with this diversity of function, the regulation and initiation of apoptosis is an intricately regulated process, which is achieved through extensive protein-protein interactions. Both the death-inducing signaling complex (DISC) and the apoptosome contain components that use the death domain (DD), death effector domain (DED), or caspase recruitment domain (CARD) to mediate protein-protein contacts [2,3]. These three protein families have been collectively termed the death domain superfamily. These domains are generally used to activate downstream signaling, often in the form of caspase or kinase activation. Each of the three families binds through homotypic interactions, such that DD-DD, DED-

*Corresponding author. Fax: (1)-734-764 4308.

E-mail: vincenz@umich.edu
DED, and CARD-CARD interactions are thought to be exclusively formed.

Experimental data regarding the structural basis for DDDD and CARD-CARD interactions have been obtained through the two heterodimer structures of Pelle/Tube and Apaf-1/procaspase-9 [4,5]. The death domain superfamily proteins are composed of an anti-parallel six helical bundle structure in the Greek key topology, with significant variations in the length and positioning of secondary structure elements [6]. The complex between Apaf-1 and procaspase- 9 involves interactions between faces of the protein formed chiefly by helices 2 and 3 and helices 1 and 4, respectively (termed a type I interaction) [4]. In contrast, the complex between Pelle and Tube chiefly involves the loops between helices 1 and 2, 4 and 5 , and 5 and 6 (type II interaction) [5]. Thus, the prevailing view is that the DD family and the CARD family use fundamentally different heterodimerization modes.

\section{Materials and methods}

The sequence similarity across the death domain superfamily is low, making it difficult to align the sequences based on the primary structure alone. We therefore decided to attempt to rigorously align the three divergent family members using a two step structure-based sequence alignment method. In the first step, each of the three families that comprise the death domain superfamily, DD, DED, and CARD, were iteratively aligned using Psi-BLAST. In the second step, the 10 death domain superfamily members of which the structures are known were superimposed upon $\mathrm{p} 75^{\mathrm{NTR}}$ using DALI [7]. The DALI alignments take into account the tertiary structure, including topological considerations. The DALI superpositions were manually evaluated and optimized, which involved minimizing discrepancies between family members, by calculating the nearest neighbor of each $\mathrm{C}_{\alpha}$ atom of p $75^{\mathrm{NTR}}$ to the nine other structures. These alignments form the basis for subsequent superposition calculations.

Superpositions were performed using linear least squares methods as implemented in the program $\mathrm{O}$ [8]. The basis of the superpositions was determined from the DALI alignments, in which only the $C_{\alpha}$ atoms of the homologous residues were used to calculate the rotation and translation matrices. The initial arrangement of the hexamer was determined from a serial superposition of the Apaf-1/procaspase- 9 and Pelle/Tube heterodimers. The P1:T1 heterodimer of the two Pelle/Tube heterodimers found in the asymmetric unit cell was chosen. For example, Pelle and Apaf-1 were superimposed, which resulted in the contiguous placement of three death domain superfamily members: Tube, procaspase- 9 and the superimposed structures of Pelle plus Apaf-1. Either one of the superimposed structures (Pelle or Apaf-1) is removed, leaving a trimer in which the individual domains interact via type I (Apaf-1/procaspase-9), type II (Pelle/Tube), and the newly formed type III interface. Such superpositions of one member of the heterodimer structures onto a member of the growing multimer are repeated until the heterohexamer is formed. In the final step, Fas 
and FADD were superimposed onto the members of the hexamer (Pelle, Tube, Apaf-1, and procaspase-9).

Interaction interfaces were evaluated based primarily upon electrostatic compatibility. The interaction faces were inspected for electrostatic compatibility by enumerating residues that are $6 \mathrm{~A}$ or less from each other. Precise van der Waals and electrostatic compatibility was not a primary consideration since the interfaces were not optimized using energy minimization to avoid model bias. Plasticity of the interfaces has been experimentally shown in the Pelle/Tube heterodimer in which the two heterodimers in the asymmetric unit have a $7^{\circ}$ rotation due to the insertion of a crystallographic related molecule in the interface [5].
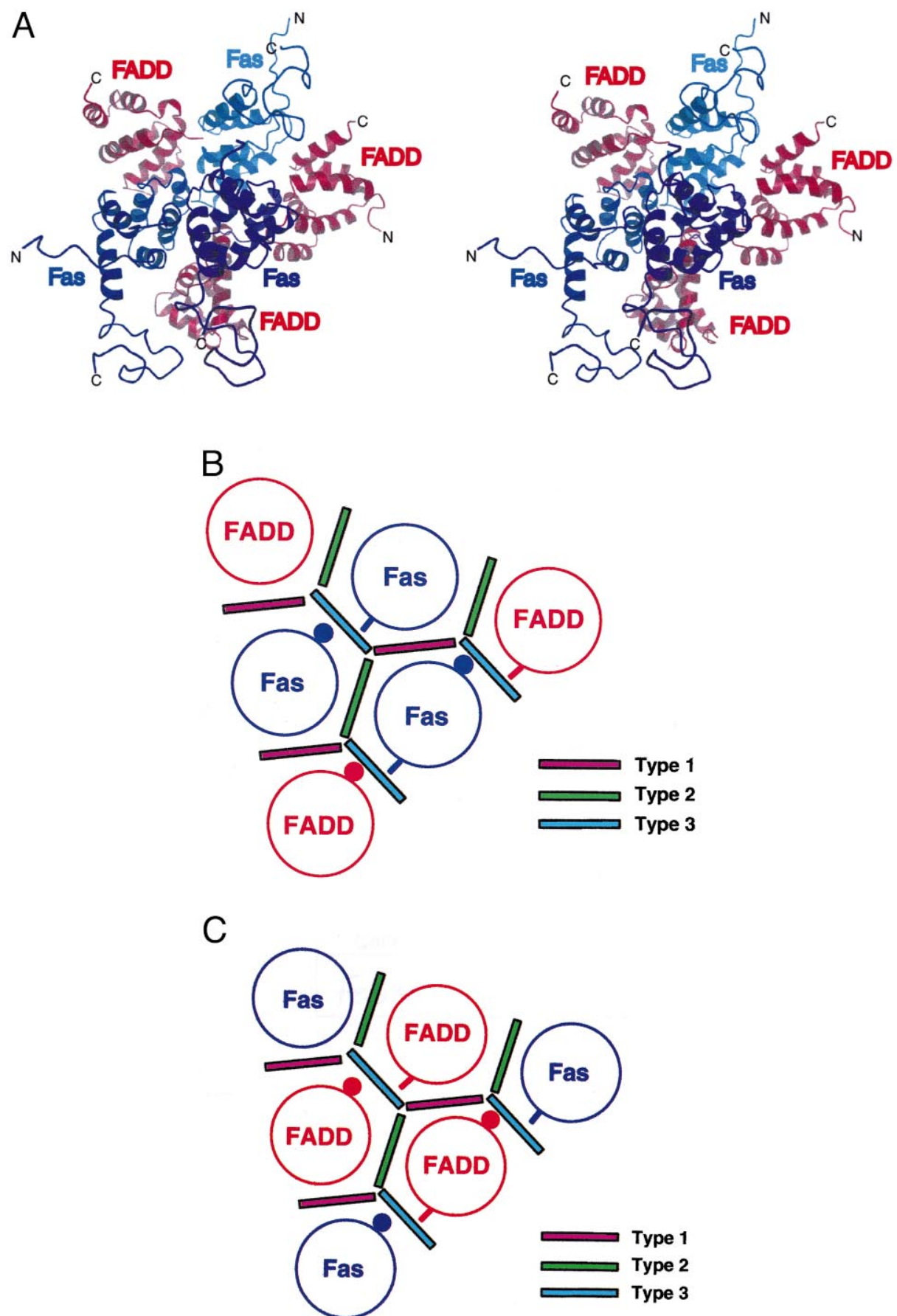

Fig. 1. Docking model of the Fas/FADD DD heterohexamer. A: Ribbon diagram illustrating the three-dimensional relationship found in the heterohexamer composed of Fas (blue) and FADD (red). The view is parallel to and facing the cell membrane. This figure was drawn using Molscript [28] and rendered using POV-Ray. B: Illustration of the three different types of interactions found in a Fas centered DISC complex viewed from the same vantage point as above. The type I interaction (magenta) corresponds to the dimer interface of the CARD of Apaf-1 and procaspase-9 [4]. The type II interface (green) corresponds to the dimer interface of the DD of Pelle and Tube [5]. The type III interaction, shown in cyan, is a novel interaction face formed as two DDs interact via the type I and II interfaces. Thus, this interface is the result of the juxtaposition of a type I and a type II interface. C: Illustration of the FADD centered DISC complex. The vantage point and color coding are identical to that in $\mathrm{B}$. 


\section{Results and discussion}

Closer examination of these death domain superfamily structures led us to the striking observation that the type I and type II interactions are not mutually exclusive and, furthermore, superposition of Pelle and procaspase- 9 results in a well packed trimer consisting of the DD of Tube, Pelle (or procaspase-9), and Apaf-1. The binding interfaces are adjacent to each other, and form a third interface (type III). These observations, obtained only through rotation and translation of existing structures, prompted this docking model study of Fas and FADD.

Fas, the receptor for Fas ligand, and FADD, an adapter molecule which links Fas to procaspase-8, interact through their DDs [9-11]. The structures of both the Fas DD and the FADD DD have been determined by nuclear magnetic resonance (NMR) [6,12]. To generate a model of the DISC, we have superimposed the DDs of Fas and FADD over the DD of Pelle and Tube, as well as the CARD of Apaf-1 and procaspase-9 using translation and rotation matrices determined from a DALI structural alignment [7]. We extended the docking model from a trimer to a hexamer to accommodate data suggesting that Fas receptor exists as a trimer and each Fas DD binds one FADD DD [13-15]. The additional molecules were positioned using the same rotation matrices calculated to form the trimer complex, thus minimizing manual intervention in the docking process [8]. The resulting model is based completely upon NMR and crystallographic data using known domain-domain interactions to dock Fas and FADD. The proposed complex is structurally and biologically sensible (Fig. 1A).

The C-terminal tails of the DDs, notably of Fas and Tube, were omitted from the sequence alignment due to a lack of significant similarity. In addition, the C-terminal tail of Fas is disordered in the NMR structure [6]. However, analogous to the C-terminal tail of Tube it is likely that the tail of Fas would interact with its partner. Nevertheless, the packing of the C-terminal tail of Tube against Pelle is unlikely to be conserved in detail in the Fas and FADD oligomer. Thus it is not possible to interpret experiments, such as those that implicate the C-terminal tail in lowering the affinity of Fas for FADD $[11,16]$, in the context of the proposed heterohexamer.

The complex consists of six DD modules bound together. The three interfaces form a repeating pattern, reminiscent of a triskelion (Fig. 1). The triskelion occurs three times in the heterohexamer and the identity of the three proteins varies among each, such that each of the three Fas and the three FADD molecules is bound in distinct environments. Each Fas and FADD molecule makes both homo- and heterodimer interactions using each of the three interfaces (Fig. 1B). The

Fig. 2. Diagram indicating residues of Fas and FADD with the potential to interact. Residues within $6 \AA$ of a neighboring molecule (which allows for side chain movement) are indicated. Cyan lines indicate possible homotypic interactions while black lines indicate possible heterotypic interactions. Generally these possible interactions are electrostatic in nature, although selected van der Waals interactions are also indicated. Residues shaded gray are found at a single interaction face only. Residues highlighted red are mutations that eliminate binding or signaling, residues in yellow have a moderate effect, and green residues do not have any effect.

A: Type I interface. B: Type II interface. C: Type III interface.
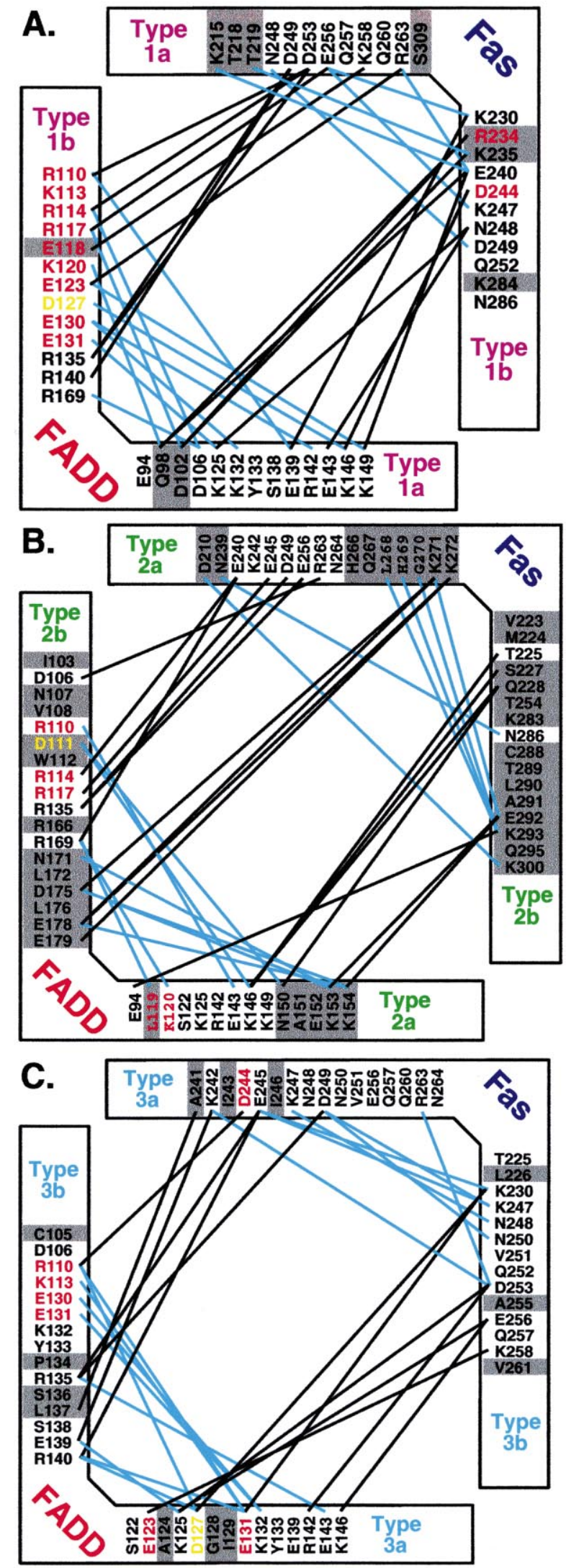
binding surfaces of Fas and FADD are complementary in both shape and electrostatic nature facilitating the formation of these varied interactions through each of the three interfaces. The interfaces are composed of a mixture of possible van der Waals interactions, hydrogen bonds, and 4-9 salt bridges. Since the interaction surfaces have not been energy minimized to avoid model bias, residues with side chains within $6 \AA$ of each other have been listed in Fig. 2. Several of these residues are specific to a single interface, however many of the amino acids listed can potentially participate in more than one type of interaction. Resolution of these ambiguities will have to await experimental verification to establish any conformational changes and induced fit. Plasticity of the interfaces has been experimentally shown in the Pelle/Tube heterodimer structure where the two dimer interfaces in the asymmetric unit differ by a $7^{\circ}$ rotation [5]. The Fas and FADD DDs fit well together, requiring only one loop, which lies between helices 3 and 4 , to move. Indeed there is precedent for this movement in the Pelle/Tube heterodimer structure in which this loop of Tube is swung out (Fig. 3) [5].

The proposed docking model is specific and excludes several interactions not observed experimentally. We have attempted to incorporate the solution structure of the FADD DED into the complex [17]. None of the six possible interfaces of either the Fas DD or FADD DD are compatible with those of FADD DED. FADD DED does not form chemically sensible contacts with any of the postulated interfaces of either FADD DD or Fas DD. The interfaces are not electrostatically complementary, resulting in significant charge-charge repulsions (unpublished data). Likewise, a heterohexamer involving either Pelle or Tube and either Apaf-1 or procaspase-9 does not result in a chemically sensible complex, as expected from biological and functional data. This underscores the uniqueness

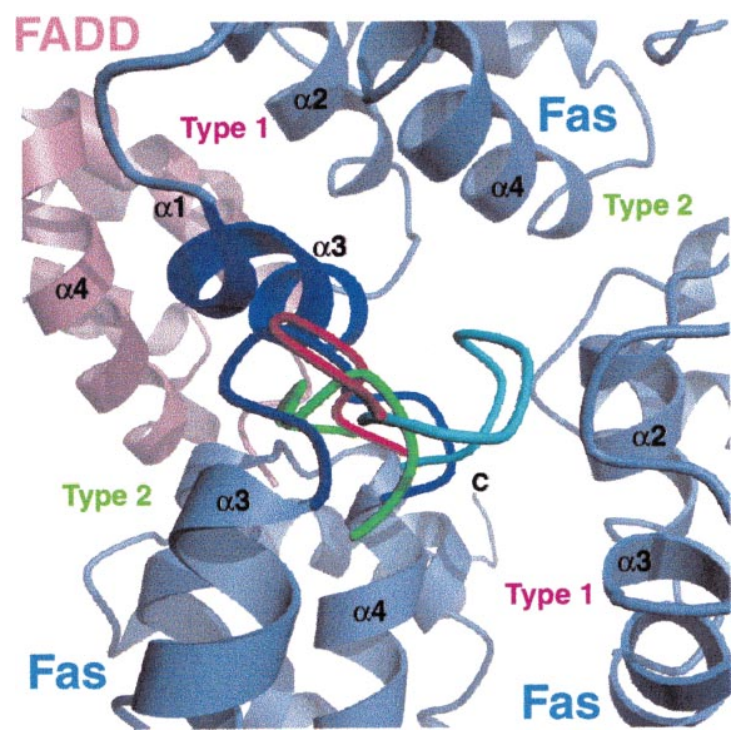

Fig. 3. The loop between helices 3 and 4 at the type III interface is mobile. This loop is displaced in Tube (cyan) relative to FADD (red), Fas (dark blue), and procaspase-9 (green) which prevents steric hindrance between this loop and helix 3 of the neighboring molecule. Three Fas DDs (light blue) and a FADD DD (red) are rendered in ribbon format. The key components of the type III interface, helix 3 of one Fas DD and the loop between helices 3 and 4 of a second Fas DD, are shown in dark blue. of the FADD/Fas complex and illustrates the specificity of all three modeled interfaces.

Two fundamentally different heterohexamers can be envisioned, one in which there is a Fas trimer at the center, surrounded by three FADD molecules, and a second, in which a FADD trimer is located at the center surrounded by three Fas molecules (Fig. 1B) [18]. Each of these models is equally probable when based upon a qualitative inspection of the interaction interfaces. The C-termini in the crystal structure of the extracellular domain of tumor necrosis factor receptor (TNFR) bound to TNF $\beta$ are relatively far apart (33 Å) [19], providing circumstantial evidence that the three FADD may be located at the center of the heterohexamer. Although the absolute stoichiometry of the members of the DISC complex has not been established, the current model is consistent with a Fas trimer interacting with three FADD molecules. Due to the degenerate nature of the interactions, the formation of larger complexes consisting of multiple trimers cannot be ruled out, although such larger aggregates have not been experimentally observed.

Published site-directed mutagenesis (SDM) data are consistent with the proposed docking model of the DISC complex (Fig. 4). Extensive SDM studies have been carried out using the DDs of human TRADD [20], TNFR [21], and Fas [2224]. In each case residues affecting binding, function or both were spread throughout the length of the protein. This result is inconsistent with the proposed interaction model of Fas and FADD, in which an anti-parallel interaction involving only helices 2 and 3 has been proposed [12].

Inactivating mutations were found along the length of the Fas DD. Fas mutations which showed binding effects are located in helices 1 through 6 , as well as between helices 1 through 5 [23]. Significantly these mutations would affect all three of the proposed interaction types. The DD of TNFR has also been mutated [21], and residues that lower activity can be found in all six secondary structure elements leading to the suggestion that TNFR uses at least two binding interfaces that are each used in homo- as well as heterodimeric interactions [24]. TRADD DD residues have been mutated sequentially to alanine 3-4 amino acid residues at a time [20]. These mutants were analyzed for binding and induction of apoptosis and inactivating mutations were found the length of the protein, not merely at a single DD interface.

Difficulties exist in interpreting these SDM data. For example, Gly 92 and Gln 93 of Pelle are involved in binding Tube, yet G92W, Q93G and Q93W show normal Tube binding activity [5]. Likewise, the TNFR mutants E390A and E406A individually show normal cytotoxic activity, yet when the double mutant is made cytotoxic activity is reduced indicating that elimination of a weak interaction does not necessarily result in a loss of function [21]. Thus, negative SDM results must be interpreted with care. In addition, residues that appear to affect binding or signaling must be regarded with care due to folding and stability effects. For example, the $l p r$ mutation in Fas (V238N) results in the local unfolding of helix 3 thereby affecting a significant number of residues beyond Val 238 [25]. Despite these concerns, SDM remains a powerful tool for the analysis of binding interfaces.

Significantly, all of the mutations that show either a full or partial effect are located near one or more of the interfaces. Due to variations in loop lengths and helix positioning a precise correlation between sequence alignments between 


\begin{tabular}{|c|c|}
\hline & $\begin{array}{c}\text { HELIX } 2 \\
\&\end{array}$ \\
\hline & 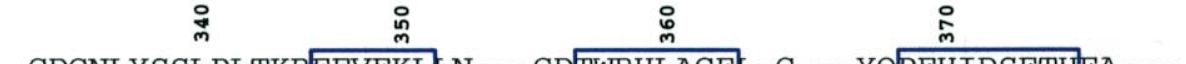 \\
\hline & GDGNLYSSLPLTKREEVEKLEN---GDEWRHLAGE-G---YQPEHIDSFTHEA-- \\
\hline D & SRNTELRRVEDNDIYRLAKILDE--NSSCWRKLMSIII $\mathrm{P}$ KK--YTAQDVFQIDEAANR- \\
\hline DD & DNTMAIRLLPEPVRAQLCAHLDAL-DVWQQLATAV--K--LY PDQVEQISSQKQR- \\
\hline & DVDLSK YITTIAGVMT---LSQVKGFVRKN-G---VN EAKIDEIKN---- \\
\hline -DD & 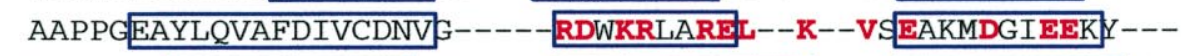 \\
\hline & AGAAPGEEDLCAAFNVICDNVG----- KDWRRLARQL--K--V: DTKIDSIEDRY--- \\
\hline h'T & -KPQSLDTDDPATLYAVVEN N---PPLRW KEFVRRL-G---LSDHEIDRLELQ---- \\
\hline & FQPVVNRPLSLKDQQTFARSV----GLKWRKVGRSL-Q---RGCRALRDPALDSLAY \\
\hline D & . MDPFLVLLHSVSSSL---SSSELTELKGLCDGR--VGKRKLERVQ------ \\
\hline RD & MDEADRRLLRR RLRLVEELQ--VDQ LWDVLLSREL----FR PHMIEDIQRAGSG- \\
\hline $\mathbf{D}$ & YQEGI----LTENHIQETNAQTT-- \\
\hline RD & MDAKARNCLLQ \\
\hline DR & MADQLLRKKRRIFTHSVG---AGTINALLDCLLEDEVISSEEDMKKVR------ \\
\hline & -Type 1a- --Type2b-- Type 1b Type 1b \\
\hline & HELIX 4 \\
\hline & ஓ् \\
\hline & --------CPVRALLASWGA $2----D S A T$ LDALLAALRRIQR ADIVESLCSESTA- \\
\hline & ---LPPDQS KSQMMIDEWKTSSGKLNERPTVGVLLQLLVQAEI ESAADFVALDFLNE \\
\hline DD & ------GRSASNEFLNIWGG ---YNHTVQTLFALFKKLKI HNAMRLIEDYVSED \\
\hline & -DNVQDTAEQKVQLLRNWHQL":--GKKE AYDTLIKDLKKANLCTLAEKIQTIILKDI \\
\hline DD & ---PRS LSERVRESLKVWKNAEK--KNASVAGLVKALRTCRL NLVADLVEEAdES- \\
\hline & ---PRNL TERVRESLRIWKNTEK--ENATVAHLVGALRSCRMNLVADLVQEVQQAR \\
\hline $\mathrm{DD}$ & --NGRCLREAQYSMLATWRRRTPRRE-ATLELLGRVLRDMDLLCCLIDIEEALCGP \\
\hline DED & EYEREGLYEQAFQLLRRFVQAEGRR--ATLQRLVEALEENELTSLAEDLIGLTDPN \\
\hline D_DED & ---------SGLDLFSMLLEQND-LEPGHTELLRELLASDRR HDLLRRVDDFELE- \\
\hline & R----GSQ $\overline{\text { ALPLFISCLFDTGQ }}$ \\
\hline סגר & -----GLRKTMLLLDTLPSR---GPK-A EDTFLDSLQEEPWVREKLKKAREEAMT \\
\hline & $-----T$ QQQRAAMLIKMIDKK----D NDSYVSFYNALLHE----GY KDLAALDHDG \\
\hline berg_CARD & 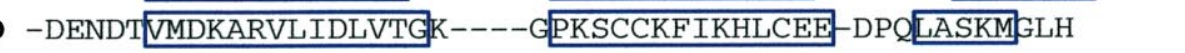 \\
\hline & a- -Tyре2a- \\
\hline
\end{tabular}

Fig. 4. Structure-based sequence alignment of the death domain superfamily. Residues shown by X-ray crystallography to be involved in heterodimerization are indicated by magenta circles below the sequence [4,5]. Residues highlighted in red have been shown by SDM to be involved in binding or signaling, those shown in yellow show an effect when more than one residue is mutated at a time, those indicated in green show no effect. Blue boxes indicate helices, while proteins named in bold have been structurally characterized. The secondary structure of TNFR has been assigned using NMR, however a complete structure is unavailable at this time [24]. The green asterisk indicates a two helix insert, omitted for clarity, found only in Tube. The C-terminal tail has been omitted due to the low level of sequence homology, although a large number of death domain superfamily members possess a C-terminal extension, including Fas, FADD, and DAP kinase. Structural and mutational references: p $75^{\mathrm{NTR}}$ [29], Pelle and Tube [5], Fas [6], FADD [12,17], TNFR [24], TRADD [20], caspase-9 and Apaf-1 [4], RAIDD [30], and Iceberg [31].

proteins with unknown structures and the experimentally determined heterodimer structures is not expected. The significance of the accumulated SDM data lies in the extended pattern of affected residues observed in TNFR, Fas, and TRADD, affecting the length of the DD.

The docking model is testable using targeted SDM initially, culminating in the crystallographic structure determination of the complex. Critical to a SDM analysis, several residues are predicted to make interactions specific to one interface only, especially in the type I and II interfaces, permitting targeted investigation (Fig. 2). Specifically, Lys 215, which is postulated to interact with Glu 240 in a type I Fas-Fas DD interaction, is unique and can be studied (Fig. 2A). The K215E and $\mathrm{E} 240 \mathrm{~K}$ mutants can be studied for both individual reduction in binding as well as in complementation studies. The type I interface specific to heterodimer formation can be in- 
vestigated through mutations of Asp 102 of FADD, which is postulated to interact with either Arg 134 or Lys 235 of Fas, both of which are only involved in the type I interaction. The other two interfaces can be studied through analogous mutations (Fig. 2). An exception is the narrow type III interface consisting mainly of helix 3 , which is isolated from the majority of the six helix bundle. Residues of this interface therefore are difficult to unambiguously identify at this point, with the exception of selected hydrophobic residues. It is unclear whether conformational changes, either transmitted from the extracellular domain of Fas or due to DD binding, alter the character of this binding surface. Flexibility in helix 3 has been noted in several NMR studies of DDs [12,24].

The docking model is not only consistent with published SDM data, but also explains diverse phenomena. This hypothesis is needed due to the SDM evidence suggesting that multivalent interactions are common in the death domain superfamily. Tight association of the three Fas molecules may facilitate the transmission of conformational changes from the extracellular portion of the receptor that binds Fas ligand. It has recently been shown that the Fas trimer exists in the absence of Fas ligand binding [15]. This implies that conformational changes must be transferred upon ligand binding. Indeed, it has been shown that a conformational change occurs upon Fas activation using chemical crosslinking experiments [15].

Death effector filaments (DEFs), which are formed by some death domain superfamily members, are extensive, elongated aggregates observed upon overexpression [26]. The formation of DEFs implies that the death domain superfamily forms multivalent and simultaneous interactions consistent with our model. DEFs may form through the preferential binding of monomers to a particular surface, thus forming elongated fibers.

Heterohexamer structures have not been observed in crystallographic analyses so far. However, several of the studied molecules have been altered to optimize solubility [17]. These changes required to obtain heterodimers and avoid aggregation may also have inadvertently eliminated multivalent interaction modes. The C-terminal tail may be indispensable for complex formation as indicated by its pronounced involvement in the type II interface [5]. In addition, full length molecules may be required for complex formation. Full length FADD, containing both the DD and DED, has been shown to self associate by yeast two hybrid studies, yet the isolated FADD DD does not [10,27].

This docking model opens new avenues of study regarding potential interactions between members of the apoptotic signaling machinery. Further Fas and FADD site-directed mutants will have to be generated and quantitative binding studies will have to be performed in order to further substantiate this model. We believe this docking model extends the current monovalent models of death domain superfamily interactions and is more consistent with existing SDM data. It will be interesting to investigate other large signaling complexes such as the apoptosome to determine if similar binding patterns are found.

\section{References}

[1] Thompson, C.B. (1995) Science 267, 1456-1462.

[2] Aravind, L., Dixit, V.M. and Koonin, E.V. (1999) Trends Biochem. Sci. 24, 47-53.

[3] Kischkel, F.C., Hellbardt, S., Behrmann, I., Germer, M., Pawlita, M., Krammer, P.H. and Peter, M.E. (1995) EMBO J. 14, 5579-5588.

[4] Qin, H., Srinivasula, S.M., Wu, G., Fernandes-Alnemri, T., Alnemri, E.S. and Shi, Y. (1999) Nature 399, 549-557.

[5] Xiao, T., Towb, P., Wasserman, S.A. and Sprang, S.R. (1999) Cell 99, 545-555.

[6] Huang, B., Eberstadt, M., Olejniczak, E.T., Meadows, R.P. and Fesik, S.W. (1996) Nature 384, 638-641.

[7] Holm, L. and Sander, C. (1993) J. Mol. Biol. 233, 123-138.

[8] Jones, T.A. and Kjeldgaard, M. (1993) Uppsala University, Uppsala, Sweden.

[9] Boldin, M.P., Goncharov, T.M., Goltsev, Y.V. and Wallach, D. (1996) Cell 85, 803-815.

[10] Boldin, M.P., Mett, I.L., Varfolomeev, E.E., Chumakov, I., Shemer-Avni, Y., Camonis, J.H. and Wallach, D. (1995) J. Biol. Chem. 270, 387-391.

[11] Chinnaiyan, A.M., O'Rourke, K., Tewari, M. and Dixit, V.M. (1995) Cell 81, 505-512.

[12] Jeong, E.J., Bang, S., Lee, T.H., Park, Y.I., Sim, W.S. and Kim, K.S. (1999) J. Biol. Chem. 274, 16337-16342.

[13] Starling, G.C., Bajorath, J., Emswiler, J., Ledbetter, J.A., Aruffo, A. and Kiener, P.A. (1997) J. Exp. Med. 185, 1487-1492.

[14] Orlinick, J.R., Vaishnaw, A., Elkon, K.B. and Chao, M.V. (1997) J. Biol. Chem. 272, 28889-28894.

[15] Siegel, R.M., Frederiksen, J.K., Zacharias, D.A., Chan, F.K., Johnson, M., Lynch, D., Tsien, R.Y. and Lenardo, M.J. (2000) Science 288, 2354-2357.

[16] Itoh, N. and Nagata, S. (1993) J. Biol. Chem. 268, 10932-10937.

[17] Eberstadt, M., Huang, B., Chen, Z., Meadows, R.P., Ng, S.C., Zheng, L., Lenardo, M.J. and Fesik, S.W. (1998) Nature 392, 941-945.

[18] Berglund, H., Olerenshaw, D., Sankar, A., Federwisch, M., McDonald, N.Q. and Driscoll, P.C. (2000) J. Mol. Biol. 302, 171-188.

[19] Banner, D.W., D’Arcy, A., Janes, W., Gentz, R., Schoenfeld, H.J., Broger, C., Loetscher, H. and Lesslauer, W. (1993) Cell 73, 431-445.

[20] Park, A. and Baichwal, V.R. (1996) J. Biol. Chem. 271, 98589862.

[21] Tartaglia, L.A., Ayres, T.M., Wong, G.H. and Goeddel, D.V. (1993) Cell 74, 845-853.

[22] Vaishnaw, A.K., Orlinick, J.R., Chu, J.L., Krammer, P.H., Chao, M.V. and Elkon, K.B. (1999) J. Clin. Invest. 103, 355-363.

[23] Martin, D.A. et al. (1999) Proc. Natl. Acad. Sci. USA 96, 45524557.

[24] Telliez, J.B. et al. (2000) J. Mol. Biol. 300, 1323-1333.

[25] Eberstadt, M., Huang, B., Olejniczak, E.T. and Fesik, S.W. (1997) Nat. Struct. Biol. 4, 983-985.

[26] Siegel, R.M., Martin, D.A., Zheng, L., Ng, S.Y., Bertin, J., Cohen, J. and Lenardo, M.J. (1998) J. Cell. Biol. 141, 1243-1253.

[27] Boldin, M.P., Varfolomeev, E.E., Pancer, Z., Mett, I.L., Camonis, J.H. and Wallach, D. (1995) J. Biol. Chem. 270, 7795-7798.

[28] Kraulis, P.J. (1991) J. Appl. Crystallogr. 24, 946-950.

[29] Liepinsh, E., Ilag, L.L., Otting, G. and Ibanez, C.F. (1997) EMBO J. 16, 4999-5005.

[30] Chou, J.J., Matsuo, H., Duan, H. and Wagner, G. (1998) Cell 94, $171-180$.

[31] Humke, E.W., Shriver, S.K., Starovasnik, M.A., Fairbrother, W.J. and Dixit, V.M. (2000) Cell 103, 99-111. 